\title{
Embryonic self-fracking
}

\author{
Marino Arroyo ${ }^{1,2}$ and Xavier Trepat ${ }^{1,3,4,5}$
}

${ }^{1}$ Institute for Bioengineering of Catalonia (IBEC), The Barcelona Institute for Science and Technology (BIST), Barcelona, Spain

${ }^{2}$ Universitat Politècnica de Catalunya-BarcelonaTech, Barcelona, Spain.

${ }^{3}$ Institució Catalana de Recerca i Estudis Avançats (ICREA), Barcelona, Spain.

${ }^{4}$ University of Barcelona, Barcelona, Spain

${ }^{5}$ Centro de Investigación Biomédica en Red en Bioingeniería, Biomateriales y Nanomedicina, Barcelona, Spain

Email: marino.arroyo@upc.edu, xtrepat@ibecbarcelona.eu

From a broken bone to a major earthquake, the fracture of a material usually means trouble. In some applications, however, humans have learned to use fracture in their own benefit. This is well illustrated by the process of hydraulic fracturing or 'fracking', whereby injection of pressurized fluid in shale rocks opens cracks to extract oil or gas (1). On page XXX of this issue, Dumortier et al show that the mouse embryo uses this very same principle to transiently disrupt its shape and sculpt a new one (2). Unlike fracking of sedimentary rocks, which causes irreversible cracking and may result in severe ecological damage, embryonic fracking is exquisite; a new embryonic structure emerges and no damage is left behind.

During the first days of development, the pre-implantation mammalian embryo is a cell aggregate that undergoes rounds of division while remaining compact and radially symmetric (Fig. 1a). Radial symmetry is first broken when the embryo becomes a blastocyst -a structure composed of a fluid-filled lumen called the blastocoel, an enveloping cellular layer called the trophectoderm, and an inner cell mass that contains embryonic progenitors (3) (Fig. 1d). As development proceeds, the trophectoderm will give rise to the placenta, while the inner cell mass progenitors will give rise to the entire fetus. Over the past decade, different types of single cell 'omics' have provided an increasingly detailed description of the molecular changes that accompany symmetry breaking during the formation of the blastocyst (4). However, a structural symmetry breaking is ultimately a mechanical event, and no amount of 'omic' detail will ever suffice to understand this event unless integrated into a mechanical framework.

To provide such framework, Dumortier et al performed high resolution life imaging of the formation of the blastocyst. Strikingly, after the fifth round of division, they observed the synchronous appearance of hundreds of bubbles at cell-cell junctions (Fig. 1b). These bubbles 
were filled with pressurized water and their formation was impaired by disruption of cell polarity, reversion of the osmotic gradient, and inhibition of ion transport through $\mathrm{Na} / \mathrm{K}$ pumps. During bubble formation, the main cell-cell adhesion protein of the blastocyst (E-cadherin) reorganized its spatial distribution to accumulate at the edges of the bubbles (Fig. 1e). Together, these experiments provide solid evidence that bubbles form through hydraulic fracturing: pressurized fluid is injected between two previously cohesive cell membranes, causing their separation and the displacement of cell-cell adhesion proteins. Similar pressurized fluid bubbles disrupting cell-cell and cell-matrix interfaces had been reported in vitro as a result of poroelastic flows (5), osmotic perturbations (6), or directed ionic transport (7). Dumortier et al now provide evidence of hydraulic fractures of cell-cell junctions in vivo.

The authors then asked how the embryo implements the next step in its morphogenetic plan, transitioning from hundreds of small fluid-filled microlumens at all cell-cell junctions to a single large lumen, the blastocoel. To understand this process, they followed the dynamics of the microlumens and found that, following a homogeneous growth phase, some microlumens started growing at the expense of others until a single lumen took over the rest (Fig. 1c). The blastocoel thus emerged from the coarsening of fluid droplets enclosed by surface tension (here provided by the cell cortex) in a process reminiscent of Ostwald ripening. Ostwald ripening is a prominent physical mechanism explaining a variety of coarsening phenomena ranging from crystal growth to the breaking of your salad vinaigrette (8). It proceeds by diffusive mass transport from the smaller droplets, which experience higher pressure following Laplace's law, towards larger ones (Fig. 1f). The observations by Dumortier et al support an analogous physical picture, with the exception that in the mouse embryo mass transport between lumens proceeds by fluid flow through a hydraulically connected network of intercellular gaps rather than by diffusion.

These largely physical and unspecific mechanisms of hydraulic fracturing and coarsening, however, did not explain the precise positioning of the blastocoel at the interphase between the trophectoderm and the inner mass cells. To address this question, the authors developed a theoretical model and tested its predictions using chimeric mutant embryos. By patterning stickiness and contractility, they were able to direct microlumen coarsening towards less adhesive and less contractile cell-cell junctions. Since in wild type embryos they could find evidence of contractility patterning, but not of adhesive patterning, they concluded that blastocoel positioning was guided by contractility (Fig 1c,f). Dumortier et al thus propose that the blastocoel is robustly positioned by hydraulically opening potential lumens throughout the embryo, and then delicately guiding the coarsening process by differences in cell contractility, in an intriguing interplay between physical and biological patterning.

By identifying the role of hydromechanics during early development, this study raises a wide set of questions. From a general perspective, one is compelled to ask whether profuse fracking followed by coarsening is a general mechanism of luminogenesis (9). From a mechanistic perspective, the water required to open microlumens or to swell the blastocoel should be 
driven by an actively maintained osmotic imbalance and its flow rate controlled by the hydraulic resistance of water barriers. Beyond this general framework, however, the precise mechanisms determining the remarkable synchrony of microlumen opening across the embryo and the path of water flow between compartments remain unclear. A complete picture of embryonic water management will certainly require understanding the integration of biological mechanisms, such as the sealing of tight junctions or the activation of ionic pumps, and physical ones. For instance, the path of water flow across the embryo and, eventually, the breaking of radial symmetry, will depend not only on osmotic imbalances but also on the relative hydraulic resistance of tight junctions, the junctional network, and cell membranes. While Dumortier et al showed remarkable control of blastocoel positioning by tuning adhesion and contractility, future studies may attempt to direct morphogenesis by tuning hydraulic resistance or ionic transport.

The paper highlights the importance of water transport as a morphogenetic driving force. Current understanding emphasizes that embryonic shape emerges from the differential regulation of cell growth, contraction and adhesion (10). These are indeed important mechanical factors. However, by virtue of its incompressibility and of the diverse mechanisms available to transport it, water is emerging as a powerful tool for embryos to sculpt their shape. Besides direct mechanical action, fluid pressure can trigger mechanotransduction feedback, providing an additional layer of control between embryonic growth and hydraulics (11).

At a first glance, morphogenesis and fracture would seem incompatible concepts; one is all about construction and the other about destruction. The work by Dumortier et al reconcile these two concepts by showing that embryos have evolved mechanisms to exquisitely selffracture and self-heal with the ultimate goal of creating new functional shapes. The physical principles used by embryos to control hydraulic fracture are rather universal and, thus, could inspire applications outside the biological context to design new materials or improve engineering processes (12).

\section{Figure caption}

To establish its first symmetry axis (a-d), the pre-implantation mouse embryo first self-fractures profusely by pressurizing fluid in the junctional network (b), which requires fluid flow and reorganization of adhesion molecules (e). Then the embryo precisely directs an otherwise spontaneous coarsening of microlumens by spatially modulating cortical surface tension $(c, f)$.

\section{References}

1. M. B. Smith, C. Montgomery, Hydraulic fracturing. (Crc Press, 2015).

2. Dumortier, Science, (2019). 
3. Q. Chen, J. Shi, Y. Tao, M. Zernicka-Goetz, Tracing the origin of heterogeneity and symmetry breaking in the early mammalian embryo. Nat Commun 9, 1819 (2018).

4. Z. Xue et al., Genetic programs in human and mouse early embryos revealed by single-cell RNA sequencing. Nature 500, 593-597 (2013).

5. L. Casares et al., Hydraulic fracture during epithelial stretching. Nature Materials 14, 343-351 (2015).

6. C. E. Morris, J. A. Wang, V. S. Markin, The invagination of excess surface area by shrinking neurons. Biophys. J. 85, 223-235 (2003).

7. E. Latorre et al., Active superelasticity in three-dimensional epithelia of controlled shape. Nature 563, 203-208 (2018).

8. W. Ostwald, Über die vermeintliche Isomerie des roten und gelben Quecksilberoxyds und die Oberflächenspannung fester Körper. Zeitschrift für physikalische Chemie 34, 495-503 (1900).

9. S. Sigurbjornsdottir, R. Mathew, M. Leptin, Molecular mechanisms of de novo lumen formation. Nat. Rev. Mol. Cell Biol. 15, 665-676 (2014).

10. C. P. Heisenberg, Y. Bellaiche, Forces in tissue morphogenesis and patterning. Cell 153, 948-962 (2013).

11. C. J. Chan et al., Hydraulic control of mammalian embryo size and cell fate. Nature, (2019).

12. A. Lucantonio, G. Noselli, X. Trepat, A. DeSimone, M. Arroyo, Hydraulic Fracture and Toughening of a Brittle Layer Bonded to a Hydrogel. Phys. Rev. Lett. 115, 188105 (2015).

\section{Acknowledgments}

The authors are supported by the European Research Council (CoG-616480 to XT, CoG-681434 to MA), European Union (H2020-FETPROACT-01-2016-731957), Generalitat de Catalunya and CERCA program, "ICREA Academia" award (MA), the Spanish Ministry for Science and Innovation/FEDER, Obra Social "La Caixa (XT)", and Severo Ochoa Award (XT). 
a

b

Radially symmetric embryo

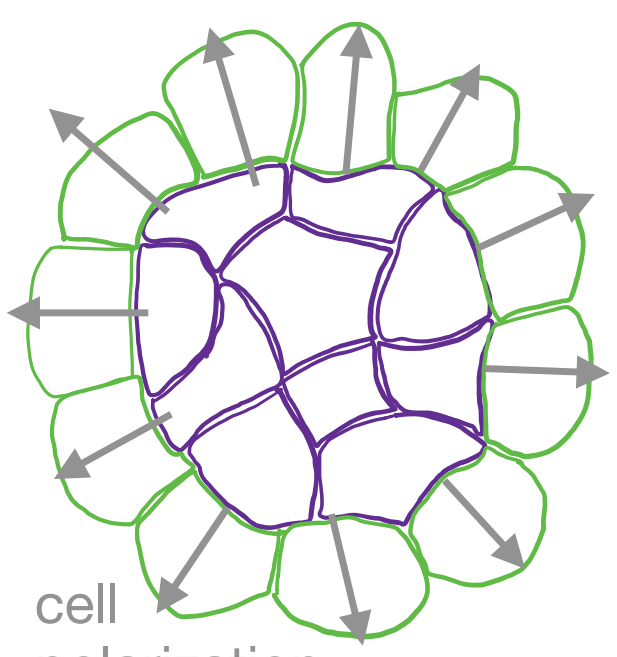

polarization

tight junctions Inner cell mass trophectoderm Fluid
Profuse fracking

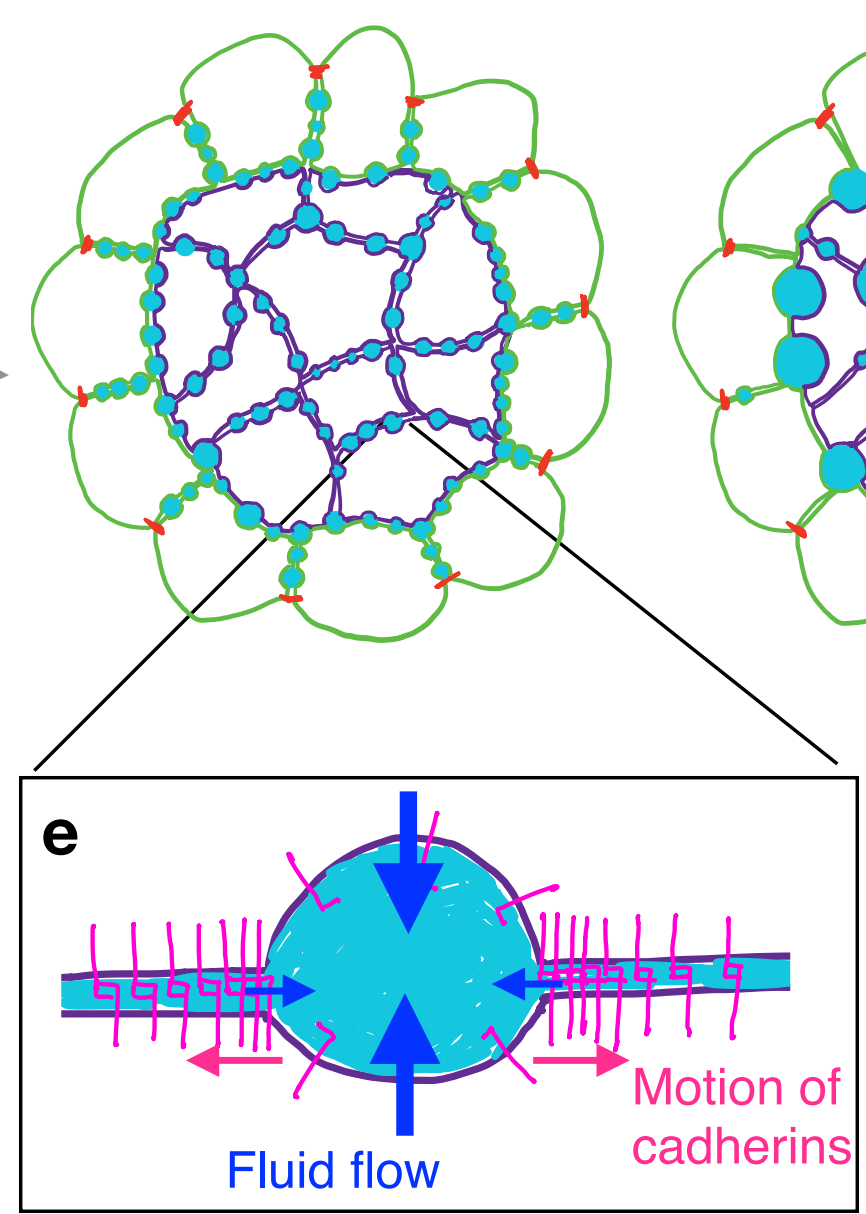

Growing microlumen
C

Directed coarsening

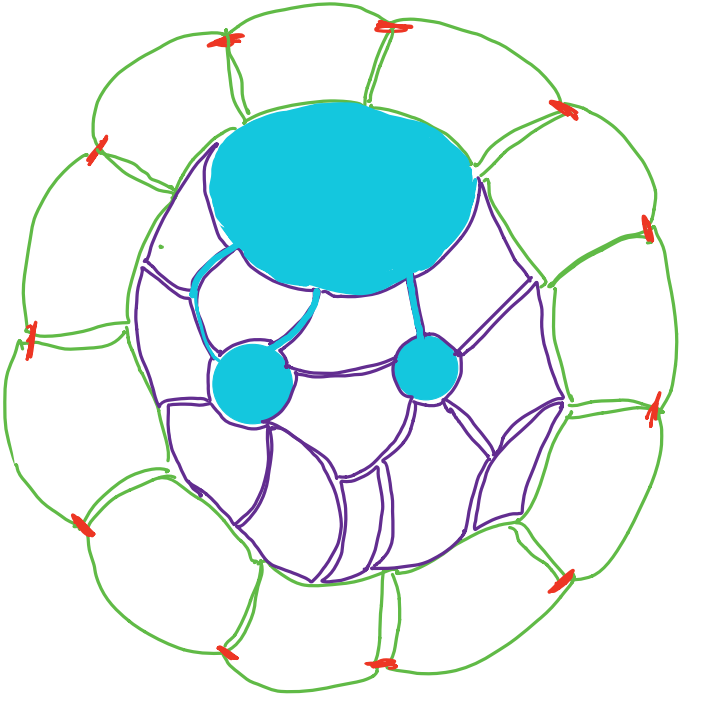

f surface tension

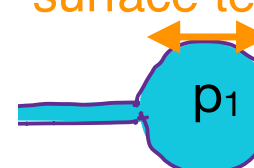

$p_{2}$

Fluid flow

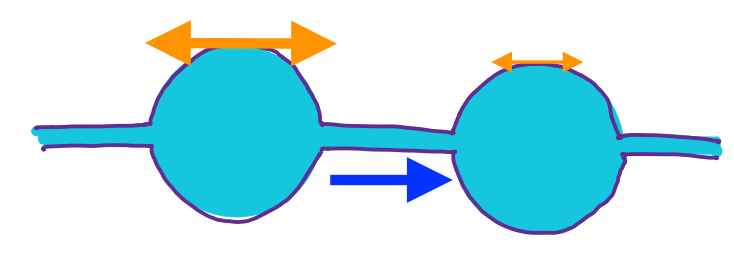

d Establishment of first symmetry axis

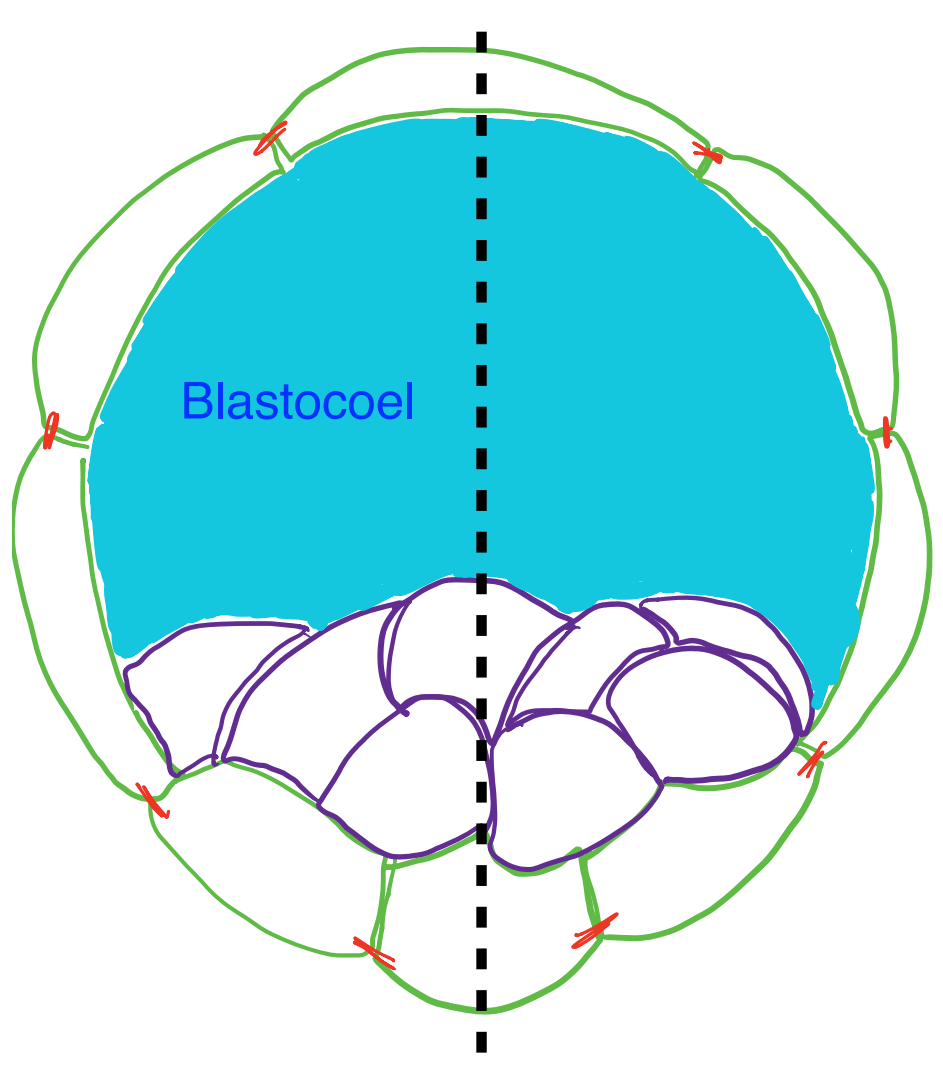

[Please, add a blue (fluid) background to panels a to $d$ around the embryo. This background should also be visible in the thin gaps between cells.] 